\title{
Intensified chemotherapy with ACVBP plus rituximab versus standard CHOP plus rituximab for the treatment of diffuse large B-cell lymphoma (LNH03-2B): an open-label randomised phase 3 trial
}

Christian Récher, Bertrand Coiffier, Corinne Haioun, Thierry Jo Molina, Christophe Fermé, Olivier Casasnovas, Catherine Thiéblemont, André Bosly,
Guy Laurent, Franck Morschhauser, Hervé Ghesquières, Fabrice Jardin, Serge Bologna, Christophe Fruchart, Bernadette Corront, Jean Gabarre,
Christophe Bonnet, Maud Janvier, Danielle Canioni, Jean-Philippe Jais, Gilles Salles, HervéTilly, for the Groupe d'Etude des Lymphomes de l'Adulte

\section{Summary}

Lancet 2011; 378: 1858-67

See Comment page 1828

Centre Hospitalier Universitaire

de Toulouse, France

(Prof C Récher MD,

Prof G Laurent MD);

Hospices Civils de Lyon,

Université Lyon-1, France

(Prof B Coiffier MD,

Prof G Salles MD); Hôpital Henri

Mondor, AP-HP, Créteil, France (Prof C Haioun MD); Université

Paris Descartes, Hôtel-Dieu,

AP-HP, Paris, France

(Prof T J Molina MD); Institut de

Cancérologie Gustave Roussy,

Villejuif, France (C Fermé MD);

Centre Hospitalier Universitaire

de Dijon, France

(O Casasnovas MD); Hôpital Saint-Louis-IUH-INSERM U728, Paris, France

(Prof C Thiéblemont MD); Université Catholique de

Louvain, Mont-Godinne,

Belgium (Prof A Bosly MD); Centre Hospitalier Universitaire de Lille, Lille, France

(F Morschhauser MD); Centre

Léon Bérard, Lyon, France

(H Guesquières MD); Centre

Henri-Becquerel, UMR918,

Université de Rouen, Rouen,

France (Prof F Jardin MD,

Prof HTilly MD); Centre

Hospitalier Universitaire de Nancy, Vandoeuvre les Nancy,

France (S Bologna MD); Centre

François Baclesse, Caen, France

(C Fruchart MD); Centre

Hospitalier d'Annecy, Pringy, France (B Corront MD); Hôpital Pitié-Salpétrière, AP-HP, Paris,

France (J Gabarre MD); Centre

Hospitalier de Liège, Liège,

Belgium (C Bonnet MD);

Institut Curie, Hôpital René

Huguenin, Saint-Cloud, France

(M Janvier MD); and Université

Paris Descartes, Hôpital Necker,

AP-HP, Paris, France

(Prof D Canioni MD, J-P Jais MD)

Background The outcome of diffuse large B-cell lymphoma has been substantially improved by the addition of the anti-CD20 monoclonal antibody rituximab to chemotherapy regimens. We aimed to assess, in patients aged 18-59 years, the potential survival benefit provided by a dose-intensive immunochemotherapy regimen plus rituximab compared with standard treatment plus rituximab.

Methods We did an open-label randomised trial comparing dose-intensive rituximab, doxorubicin, cyclophosphamide vindesine, bleomycin, and prednisone (R-ACVBP) with subsequent consolidation versus standard rituximab doxorubicin, cyclophosphamide, vincristine, and prednisone (R-CHOP). Random assignment was done with a computer-assisted randomisation-allocation sequence with a block size of four. Patients were aged 18-59 years with untreated diffuse large B-cell lymphoma and an age-adjusted international prognostic index equal to 1 . Our primary endpoint was event-free survival. Our analyses of efficacy and safety were of the intention-to-treat population. This study is registered with ClinicalTrials.gov, number NCT00140595.

Findings One patient withdrew consent before treatment and 54 did not complete treatment. After a median follow-up of 44 months, our 3-year estimate of event-free survival was $81 \%$ (95\% CI 75-86) in the R-ACVBP group and 67\% (59-73) in the R-CHOP group (hazard ratio [HR] 0.56, 95\% CI 0.38-0.83; $\mathrm{p}=0.0035$ ). 3-year estimates of progression-free survival (87\% [95\% CI, 81-91] vs 73\% [66-79]; HR 0.48 [0 - 30-0 -76]; $\mathrm{p}=0.0015)$ and overall survival (92\% [87-95] vs 84\% [77-89]; HR 0.44 [0.28-0.81]; $\mathrm{p}=0.0071)$ were also increased in the R-ACVBP group. $82(42 \%)$ of 196 patients in the R-ACVBP group experienced a serious adverse event compared with 28 (15\%) of 183 in the R-CHOP group. Grade 3-4 haematological toxic effects were more common in the R-ACVBP group, with a higher proportion of patients experiencing a febrile neutropenic episode (38\% [75 of 196] vs $9 \%$ [16 of 183]).

Interpretation Compared with standard R-CHOP, intensified immunochemotherapy with R-ACVBP significantly improves survival of patients aged 18-59 years with diffuse large B-cell lymphoma with low-intermediate risk according to the International Prognostic Index. Haematological toxic effects of the intensive regimen were raised but manageable.

Funding Groupe d'Etudes des Lymphomes de l'Adulte and Amgen.

\section{Introduction}

Diffuse large B-cell lymphoma is the most common haemopoietic malignancy, accounting for $25 \%$ of all lymphoid neoplasms. ${ }^{1}$ The clinical course of diffuse large B-cell lymphoma has greatly changed over the past 10 years, since the introduction of the chimeric monoclonal anti-CD20 antibody rituximab for the treatment of patients with lymphoma. A pivotal trial done in 1998 by the Groupe d'Etude des Lymphomes de l'Adulte (GELA) showed that combining rituximab with standard cyclophosphamide, doxorubicin, vincristine, and prednisone (CHOP) chemotherapy significantly improved response, event-free survival, and overall survival compared with CHOP alone in patients older than 60 years. ${ }^{2,3}$ These favourable results have been confirmed by three additional randomised trials in patients both younger and older than 60 years with diffuse large B-cell lymphoma. ${ }^{46}$ However, despite these significant improvements, some patients are refractory to initial treatment or can relapse after a complete response. In cases where the disease is refractory to immunochemotherapy, the estimate of progression-free survival at 3 years is only $23 \%$, even with subsequent high-dose chemotherapy and autologous stem-cell transplantation. ${ }^{7}$ This situation emphasises the importance of first-line treatment when crucial issues are still unresolved, such as establishing the optimum rituximab dose and schedule as well as the optimum chemotherapy regimen to combine with rituximab.

Two randomised studies done in the so-called prerituximab era showed that the intensified doxorubicin, 
cyclophosphamide, vindesine, bleomycin, and prednisone chemotherapy regimen (ACVBP) with subsequent sequential consolidation, compared with standard CHOP, can improve the outcome both in low-risk ${ }^{8}$ and high-risk groups of patients, according to the International Prognostic Index for aggressive lymphoma. ${ }^{10}$ Similarly, the Deutsche Studiengruppe Hochmaligne Non-HodgkinLymphome $^{11}$ showed that the addition of etoposide to CHOP improves the event-free survival of patients younger than 60 years with aggressive lymphoma. However, in a subsequent study assessing the outcomes of a subgroup of patients receiving CHOP plus etoposide in combination with rituximab compared with those receiving CHOP in combination with rituximab, the advantage conferred by CHOP plus etoposide disappeared, leading to the hypothesis that rituximab could have an equalising effect on differences exhibited by chemotherapy regimens. ${ }^{5}$

To prospectively assess the role of a dose-intensive regimen in combination with rituximab, GELA in 2003 started a multicentre, phase 3, open-label, randomised trial (LNH03-2B), comparing the efficacy and safety of ACVBP plus rituximab (R-ACVBP) versus standard CHOP plus rituximab (R-CHOP) in a selected population of patients aged 18-59 years with diffuse large B-cell lymphoma.

\section{Methods}

\section{Participants}

Between December, 2003, and December, 2008, in accordance with our protocol, we did a phase 3 multicentre randomised trial in 73 haematology or oncology departments of GELA in France, Belgium, and Switzerland. Patients were eligible if they were aged 18-59 years and had untreated diffuse large B-cell lymphoma that had been diagnosed in accordance with the WHO classification..$^{12}$ We required that patients had only one adverse prognostic factor as defined by the age-adjusted International Prognostic Index ${ }^{10}$ (raised lactate dehydrogenase, Ann Arbor stage III or IV, or Eastern Cooperative Oncology Group [ECOG] performance status 2-4 $4^{13}$ ); a minimum life expectancy of 3 months; and negative HIV, hepatitis B virus, and hepatitis $\mathrm{C}$ virus serology tests (except after vaccination). Patients were not eligible if they had T-celllymphoma; CNS or meningeal involvement by lymphoma; contraindication to any drug included in the chemotherapy regimens; any serious, active disease (according to the investigator's decision); poor renal function (creatinine $>150 \mathrm{mmol} / \mathrm{L}$ ) or poor hepatic function (total bilirubin $>30 \mathrm{mmol} / \mathrm{L}$, transaminases $>2 \cdot 5$-times the maximum normal level), unless these abnormalities were related to the lymphoma; poor bonemarrow reserve as defined by a neutrophil concentration lower than $1 \cdot 5 \times 10^{9} / \mathrm{L}$ or a platelet concentration lower than $100 \times 10^{9} / \mathrm{L}$, unless related to bone-marrow infiltration; any history of cancer during the past 5 years, with the exception of non-melanoma skin tumours or stage 0 (in situ) cervical carcinoma; treatment with any investigational drug within 30 days before the planned first cycle of chemotherapy and during the study; or any history of treated or untreated indolent lymphoma.

We required patients to provide written informed consent before registration. Our study complied with all provisions of the Declaration of Helsinki and its amendments and was done in accordance with good clinical practice guidelines. Our protocol and informed consent forms were approved by the local and national institutional review boards in each participating centre and country.

\section{Randomisation and masking}

Our trial was not masked. We randomly assigned patients in a one-to-one ratio to receive R-ACVBP or R-CHOP. Investigators enrolled the participants, and assignment to treatment was done with a computer-assisted randomisation allocation sequence with a block size of four generated by a statistician centrally at the Groupe d'Etude des Lymphomes de l'Adulte-Recherche Clinique (GELARC) offices with a fax process, without the intervention of the investigators. Treatment allocation was stratified by centre.

\section{Procedures}

The extent of the disease was assessed by physical examination; relevant laboratory tests; CT of the chest, abdomen, and pelvis (although MRI was not excluded, only CT was mandated by the protocol); cerebrospinal fluid examination; bone-marrow biopsy; and other investigational procedures, depending on clinical symptoms. The stage of lymphoma was defined in accordance with the Ann Arbor classification. 18F-fluorodeoxyglucose (18F-FDG) PET was not mandated for staging or assessment of response to treatment. Tumour measurements were assessed by the treating physician or local radiologist. We defined bulky disease as any mass $10 \mathrm{~cm}$ or greater at the maximum diameter. Performance status was assessed in accordance with the ECOG scale, ${ }^{13}$ and the lactate dehydrogonase level was expressed as the ratio of the maximum value to the normal value.

A central review was done by at least two pathologists from GELA, without knowledge of the patient outcome, to confirm the diagnosis of CD20-positive diffuse large B-cell lymphoma. Tumours were classified in accordance with the WHO classification. ${ }^{12}$ Members of the reference pathology panel are listed in the acknowledgments.

Figure 1 shows the protocol outline. Our R-ACVBP regimen consisted of an induction phase of four cycles of R-ACVBP with a subsequent consolidation phase containing different treatment sequences. A cycle of R-ACVBP comprised $375 \mathrm{mg} / \mathrm{m}^{2}$ rituximab, $75 \mathrm{mg} / \mathrm{m}^{2}$ doxorubicin, and $1200 \mathrm{mg} / \mathrm{m}^{2}$ cyclophosphamide on day $1 ; 2 \mathrm{mg} / \mathrm{m}^{2}$ vindesine and $10 \mathrm{mg}$ bleomycin on days 1 and 5 ; and $60 \mathrm{mg} / \mathrm{m}^{2}$ prednisone on days $1-5$. Each cycle of R-ACVBP was repeated every 2 weeks. Granulocyte colony-stimulating factor (filgrastim) was also given subcutaneously from day 6 to 13 . A sequential consolidation was given 4 weeks after completion of the
Correspondence to: Dr Hervé Tilly, Centre Henri-Becquerel, Université de Rouen, Rue d'Amiens, 76038 Rouen, France herve.tilly@rouen.fnclcc.fr

For the LNH03-2B protocol see http://Inh03.gela.org/ 


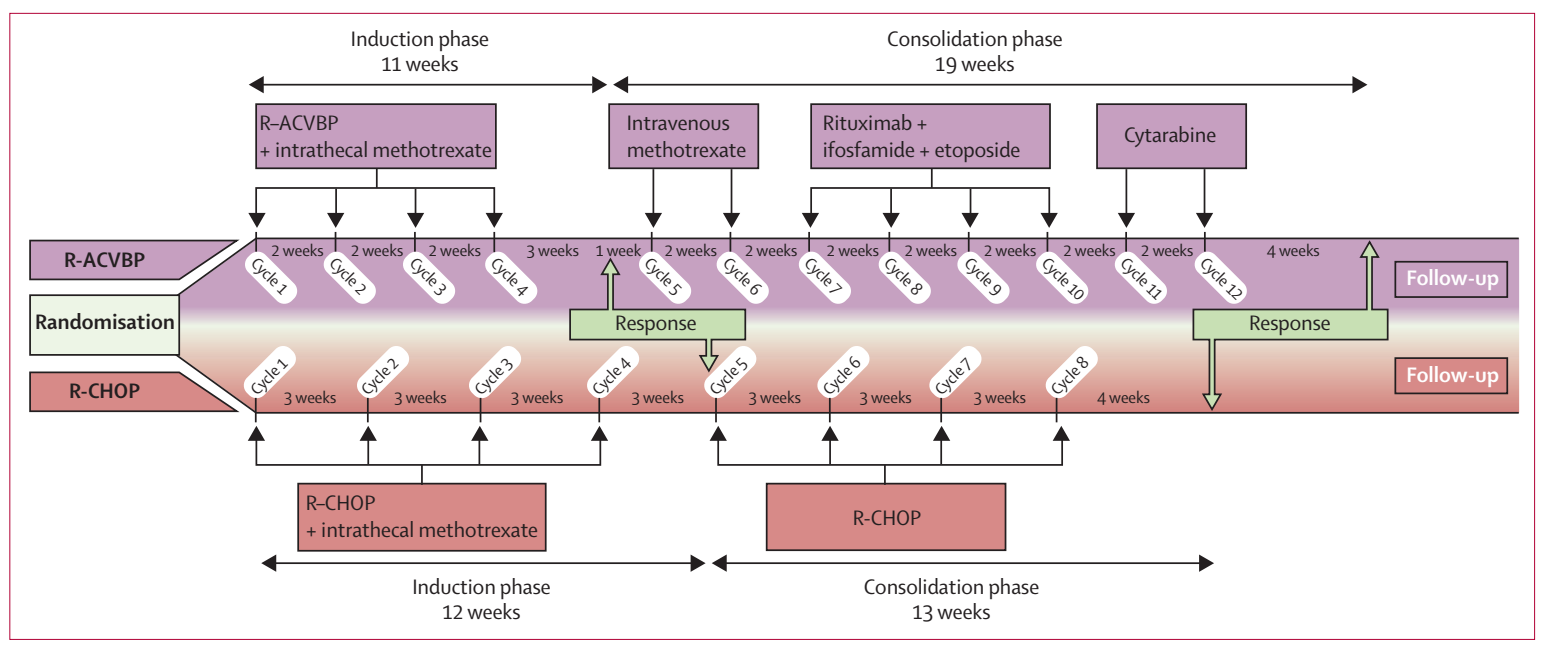

Figure 1: Protocol outline

$\mathrm{R}-\mathrm{ACVBP}=$ rituximab, doxorubicin, cyclophosphamide, vindesine, bleomycin, and prednisone. $\mathrm{R}-\mathrm{CHOP}=$ rituximab, doxorubicin, cyclophosphamide, vincristine and prednisone.

fourth cycle of R-ACVBP, consisting of two cycles of methotrexate $\left(3 \mathrm{~g} / \mathrm{m}^{2}\right)$ and calcium folinate rescue, with four subsequent cycles of rituximab $\left(375 \mathrm{mg} / \mathrm{m}^{2}\right)$ combined with etoposide $\left(300 \mathrm{mg} / \mathrm{m}^{2}\right)$ and ifosfamide $\left(1500 \mathrm{mg} / \mathrm{m}^{2}\right)$, and finally two cycles of cytarabine $\left(100 \mathrm{mg} / \mathrm{m}^{2}\right)$ given subcutaneously for 4 days, with each consolidation course being given at 2-week intervals. The R-CHOP regimen consisted of eight cycles repeated at 3-week intervals. Each cycle comprised $375 \mathrm{mg} / \mathrm{m}^{2}$ rituximab, $50 \mathrm{mg} / \mathrm{m}^{2}$ doxorubicin, $750 \mathrm{mg} / \mathrm{m}^{2}$ cyclophosphamide, and $1.4 \mathrm{mg} / \mathrm{m}^{2}$ vincristine (up to a maximum dose of $2 \mathrm{mg}$ ) on day 1 and $60 \mathrm{mg} / \mathrm{m}^{2}$ prednisone on days $1-5$. During the first four cycles, the theoretical dose intensity of the R-ACVBP regimen, compared with R-CHOP, increased by a factor of $2 \cdot 25$ for doxorubicin $\left(37.5 \mathrm{mg} / \mathrm{m}^{2}\right.$ per week vs $16.7 \mathrm{mg} / \mathrm{m}^{2}$ per week), $2 \cdot 4$ for cyclophosphamide $\left(600 \mathrm{mg} / \mathrm{m}^{2}\right.$ per week vs $250 \mathrm{mg} / \mathrm{m}^{2}$ per week), and 1.5 for rituximab $\left(187.5 \mathrm{mg} / \mathrm{m}^{2}\right.$ per week vs $125 \mathrm{mg} / \mathrm{m}^{2}$ per week). Patients received $15 \mathrm{mg}$ of intrathecal methotrexate on day 1 of the first four cycles in both study groups for prophylaxis of the CNS. Radiotherapy was not permitted in our protocol.

Tumour responses were assessed by the local investigator after four cycles of R-ACVBP and R-CHOP and at the end of treatment in each group. Responses were classified as complete response, unconfirmed complete response, or partial response. The disease state was identified as stable disease or progressive disease. Responses and disease states were based on the International Workshop 1999 criteria. ${ }^{14}$ Follow-up was undertaken by the local investigator. During the first 2 years after treatment, assessment consisted of physical examination and laboratory tests every 3 months, and CT of the chest, abdomen, and pelvis every 6 months. Thereafter, physical examination and laboratory tests were done every 6 months and CT every year.
All adverse events reported by the patient or recorded by the investigator were collected from the case report form in predefined categories. An adverse event was defined as any adverse change from the patient's baseline characteristics, independent of treatment status. Each event was graded according to the National Cancer Institute Common Toxicity Criteria grading system (version 3.0). All grade 3 and 4 events and grade 2 infections were recorded in detail. Grade 1 and 2 adverse events were not extensively described.

\section{Statistical analysis}

We used event-free survival, our primary endpoint, to assess sample size. In our previous LNH-98-5 study, ${ }^{2}$ we estimated that treatment with R-CHOP conferred a 2-year event-free survival rate of $75 \%$ in the population of patients with one factor from the age-adjusted International Prognostic Index. To detect a change in the 2-year eventfree survival from $75 \%$ to $85 \%$ in the R-ACVBP group, we calculated that 380 patients randomly assigned at a ratio of one to one between the two treatment groups, recruited over 4 years, and followed up for a minimum of 1 year, would be needed to achieve 100 events and $80 \%$ power at the overall 5\% (two-sided) significance level with a logrank test. We planned an interim analysis on the basis of 40 events after 2 years of inclusion and a follow-up of a minimum of 1 year. We used the O'Brien-Fleming $\alpha$-spending function to maintain the overall significance level, with $\alpha=0.0008$ at interim analysis and 0.05 at final analysis. Because our threshold of significance was not met at this interim analysis, we completed the second stage of our trial.

We measured event-free survival from the date of randomisation to the date of the first event. We defined events as death from any cause, disease progression during or after treatment, relapse for complete responders 
and unconfirmed complete responders, and implementation of any lymphoma treatment not stipulated by our protocol, including radiotherapy. Our secondary endpoints were response to treatment, progression-free survival, disease-free survival, overall survival, safety and the rate of CNS progression or relapse. We measured progression-free survival from the date of randomisation to the date of disease progression or relapse or death from any cause, disease-free survival from the time of attainment of a complete response or unconfirmed complete response to disease recurrence or death caused by lymphoma or treatment-related toxic effects, and overall survival from the date of randomisation to the date of death from any cause.

We computed event-free, progression-free, diseasefree, and overall survival curves by the Kaplan-Meier method and compared them by the log-rank test. We calculated hazard ratios (HR) and 95\% CIs with a Cox proportional-hazards analysis. We did univariate analyses to assess the effect of pretreatment-specified factors (age, sex, stage, number of extranodal sites, presence or absence of bone marrow involvement, presence or absence of bulky disease, B symptoms, lactate dehydrogenase concentration, $\beta 2$ microglobulin concentration, serum albumin concentration) on progression-free and overall survival. Interaction terms between these factors and treatment group assignments were also assessed with Cox analysis to detect

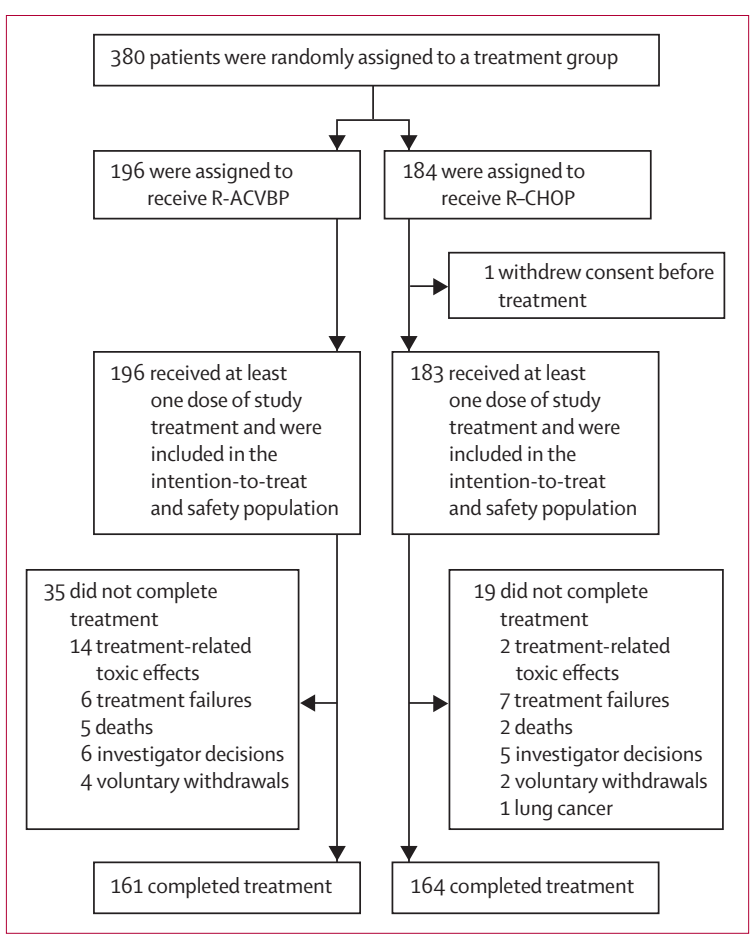

Figure 2: Trial profile

R-ACVBP=rituximab, doxorubicin, cyclophosphamide, vindesine, bleomycin, and prednisone. $\mathrm{R}-\mathrm{CHOP}=$ rituximab, doxorubicin, cyclophosphamide, vincristine, and prednisone. heterogeneity of treatment effect sizes according to the factor levels.

For each drug we calculated dose intensity as observed dose intensity divided by planned dose intensity. We expressed observed dose intensity as observed cumulative dose received during the first 4 cycles divided by observed duration between the first and fourth cycles. We defined

\begin{tabular}{|c|c|c|}
\hline & R-ACVBP group $(n=196)$ & $\mathrm{R}-\mathrm{CHOP}$ group $(\mathrm{n}=183)$ \\
\hline \multicolumn{3}{|l|}{ Sex } \\
\hline Male & $116(59 \%)$ & $109(60 \%)$ \\
\hline Female & $80(41 \%)$ & $74(40 \%)$ \\
\hline Median age, years (range, IQR) & $47(18 \cdot 0-59 \cdot 0,36 \cdot 0-54 \cdot 5)$ & $48(19 \cdot 0-59 \cdot 0,36 \cdot 0-54 \cdot 0)$ \\
\hline \multicolumn{3}{|l|}{ ECOG performance status } \\
\hline $0-1$ & $195(99 \%)$ & $182(99 \%)$ \\
\hline $2-4$ & $1(1 \%)$ & $1(1 \%)$ \\
\hline \multicolumn{3}{|l|}{ B symptoms } \\
\hline No & $137(70 \%)$ & $136(74 \%)$ \\
\hline Yes & $59(30 \%)$ & $47(26 \%)$ \\
\hline \multicolumn{3}{|l|}{ Ann Arbor stage } \\
\hline I-II & $81(41 \%)$ & $80(44 \%)$ \\
\hline III-IV & $115(59 \%)$ & $93(51 \%)$ \\
\hline \multicolumn{3}{|l|}{ Largest mass diameter $>10 \mathrm{~cm}$} \\
\hline No & $158(81 \%)$ & $137(75 \%)$ \\
\hline Yes & $38(19 \%)$ & $45(26 \%)$ \\
\hline \multicolumn{3}{|l|}{ Lactate dehydrogenase concentration } \\
\hline Normal & $119(61 \%)$ & $94(51 \%)$ \\
\hline Raised & $77(39 \%)$ & $89(49 \%)$ \\
\hline \multicolumn{3}{|l|}{ Number of extranodal sites } \\
\hline $0-1$ & $148(76 \%)$ & $133(73 \%)$ \\
\hline$>1$ & $48(24 \%)$ & $50(27 \%)$ \\
\hline \multicolumn{3}{|l|}{ Bone-marrow involvement } \\
\hline No & $165(84 \%)$ & $155(85 \%)$ \\
\hline Yes & $23(12 \%)$ & $27(15 \%)$ \\
\hline Not assessed & $8(4 \%)$ & $1(1 \%)$ \\
\hline \multicolumn{3}{|c|}{ Age-adjusted International Prognostic Index* } \\
\hline 0 & $5(3 \%)$ & $7(4 \%)$ \\
\hline 1 & $189(96 \%)$ & $169(92 \%)$ \\
\hline 2 & $2(1 \%)$ & $7(4 \%)$ \\
\hline \multicolumn{3}{|l|}{ Histology } \\
\hline Not reviewed & $23(12 \%)$ & $12(7 \%)$ \\
\hline Reviewed & $173(88 \%)$ & $171(93 \%)$ \\
\hline Diffuse large B-cell lymphoma & $156(90 \%)$ & $161(94 \%)$ \\
\hline Not diffuse large B-cell lymphoma & $13(8 \%)$ & $6(4 \%)$ \\
\hline Burkitt's lymphoma & 5 & 2 \\
\hline Follicular lymphoma & 4 & 3 \\
\hline Mantle cell lymphoma & 1 & 0 \\
\hline Marginal zone lymphoma & 1 & 1 \\
\hline Hodgkin's lymphoma & 2 & 0 \\
\hline Unclassified B-cell lymphoma & $4(2 \%)$ & $4(2 \%)$ \\
\hline \multicolumn{3}{|c|}{$\begin{array}{l}\text { Data are n (\%) unless otherwise stated. R-ACVBP=rituximab, doxorubicin, cyclophosphamide, vindesine, bleomycin, and } \\
\text { prednisone. R-CHOP=rituximab, doxorubicin, cyclophosphamide, vincristine, and prednisone. ECOG=Eastern Cooperative } \\
\text { Oncology Group. *Some included patients had an age-adjusted International Prognostic Index other than } 1 \text { at data review. }\end{array}$} \\
\hline
\end{tabular}




\begin{tabular}{|c|c|c|}
\hline & R-ACVBP group & $\mathrm{R}-\mathrm{CHOP}$ group \\
\hline Events for event-free survival & $40(20 \%)$ & $63(34 \%)$ \\
\hline Unplanned treatment for lymphoma & 15 & 14 \\
\hline Unplanned chemotherapy & 13 & 10 \\
\hline Unplanned radiotherapy & 2 & 4 \\
\hline Progression or relapse & 19 & 43 \\
\hline Death & 6 & 6 \\
\hline Events for progression-free survival & $28(14 \%)$ & $51(28 \%)$ \\
\hline Progression of relapse & 21 & 44 \\
\hline Death & 7 & 7 \\
\hline Events for overall survival & $15(8 \%)$ & $31(17 \%)$ \\
\hline Lymphoma* & 8 & 22 \\
\hline Unrelated to lymphoma progression during treatment & 5 & 3 \\
\hline \multicolumn{3}{|l|}{ Unrelated to lymphoma progression after treatment } \\
\hline Second cancer & .. & 2 \\
\hline Cardiac cause & 1 &.. \\
\hline Pneumonitis & .. & 2 \\
\hline Gastric haemorrhage & .. & 1 \\
\hline Suicide & .. & 1 \\
\hline Unknown & 1 &.. \\
\hline \multicolumn{3}{|c|}{$\begin{array}{l}\text { Data are } \mathrm{n}(\%) \text { unless otherwise stated. } \mathrm{R}-\mathrm{ACVBP}=\text { rituximab, doxorubicin, cyclophosphamide, vindesine, bleomycir } \\
\text { and prednisone. } \mathrm{R}-\mathrm{CHOP}=\text { rituximab, doxorubicin, cyclophosphamide, vincristine, and prednisone. *Deaths from } \\
\text { lymphoma progression or related to treatment of relapse or progression. }\end{array}$} \\
\hline
\end{tabular}

planned dose intensity as total planned cumulative dose received during the first 4 cycles divided by the theoretical time elapsed between the first and fourth cycles (56 days for R-ACVBP and 84 for R-CHOP).

Our analyses of efficacy and safety were of the intentionto-treat population. One patient who withdrew consent before any procedure was excluded from this population. All p values are two-sided. Statistical analyses were done with SAS 9.1.3 software by the investigators of the GELARC statistical office. This study is registered with ClinicalTrials.gov, number NCT00140595.

\section{Role of the funding source}

GELA, via GELARC, was involved in the random assignment procedure, distribution and collection of case report forms, data entry and validation, coordination of monitoring procedures, elaboration and mailing of queries, reporting of serious adverse events, coordination of histological review, relation with investigators, transmission of enrolment status to the sponsor, statistical analysis, and production of the report. Amgen had no role in study design, data collection, data analysis, data interpretation, or writing of the report. The corresponding author had full access to all the data in the study and had final responsibility for the decision to submit for publication.

\section{Results}

Figure 2 shows the trial profile. We enrolled 380 patients into our trial, of which 379 received at least one dose of the protocol-planned treatment. The main characteristics of our patients were similar in the two groups (table 1). The main criteria providing an age-adjusted International Prognostic Index score of 1 were raised lactate dehydrogenase concentration and Ann Arbor stage III-IV in $44 \%$ and $55 \%$ of patients, respectively. 21 patients had an age-adjusted International Prognostic Index score other than 1 (table 1). Central pathological review was completed for 344 patients (91\%), with diffuse large B-cell lymphoma confirmed in $90 \%$ of the R-ACVBP group and $94 \%$ of the R-CHOP group. At the data-cutoff point, Jan 1, 2010, the median follow-up was 44 months (IQR 27-53).

54 patients did not complete either treatment regimen (figure 2). 190 patients (97\%) in the R-ACVBP group received the first four cycles of the planned treatment and $177(97 \%)$ in the R-CHOP group. The median interval between these cycles was 14 days (range 12-57, IQR 14-17) in the R-ACVBP group and 21 days (14-62, 21-21) in the R-CHOP group. Based on data collected from the population who received the first four cycles of each treatment, the received dose intensities for doxorubicin were $92 \cdot 1 \%$, for cyclophosphamide were $93.0 \%$, and for rituximab were $92.3 \%$ of the planned dose for R-ACVBP, and $98 \cdot 4 \%, 98 \cdot 6 \%$, and $98 \cdot 6 \%$ for $\mathrm{R}-\mathrm{CHOP}$, respectively.

Fewer events were recorded in the R-ACVBP group than the R-CHOP group (table 2, figure 3). Event-free survival was significantly longer for patients treated with R-ACVBP than for those treated with R-CHOP $(\mathrm{p}=0.0035)$, with 3-year estimates of $81 \%$ (95\% CI 75-86) for R-ACVBP and 67\% (59-73) for R-CHOP. The HR of the R-ACVBP group for event-free survival shows that this regimen reduced the risk of experiencing an event by $44 \%$ compared with R-CHOP in this population (figure 3). Progression-free survival was significantly longer in the R-ACVBP group, resulting in a 3-year progression-free survival of $87 \%$ (81-91) with R-ACVBP and 73\% (66-79) with R-CHOP ( $\mathrm{p}=0 \cdot 0015$; figure 3$)$.

The overall response rate was $90 \%$ in the R-ACVBP group and $87 \%$ in the R-CHOP group ( $\mathrm{p}=0 \cdot 47$; table 3 ). A complete response or unconfirmed complete response was achieved in 162 patients (83\%) in the R-ACVBP group and $146(80 \%)$ in the R-CHOP group ( $\mathrm{p}=0$ - 46). In complete responders, the disease-free survival was significantly longer in the R-ACVBP group, with a 3-year estimate of $91 \%$ (95\% CI 85-95) compared with $80 \%$ (73-86) in the R-CHOP group ( $\mathrm{p}=0.0019$; figure 3 ). 15 patients in the $\mathrm{R}-\mathrm{ACVBP}$ group and 33 in the R-CHOP group experienced a relapse. There was no CNS relapse in the R-ACVBP group but two in the R-CHOP group. There were fewer deaths in the R-ACVBP group than the R-CHOP group (table 2). Overall survival differed significantly, with a 3 -year estimate of $92 \%$ (87-95) in the R-ACVBP group and $84 \%$ (77-89) in the R-CHOP group ( $\mathrm{p}=0 \cdot 0071$; figure 3 ).

Per-protocol analyses of patients who met all eligibility criteria, who had confirmed diffuse large B-cell lymphoma 


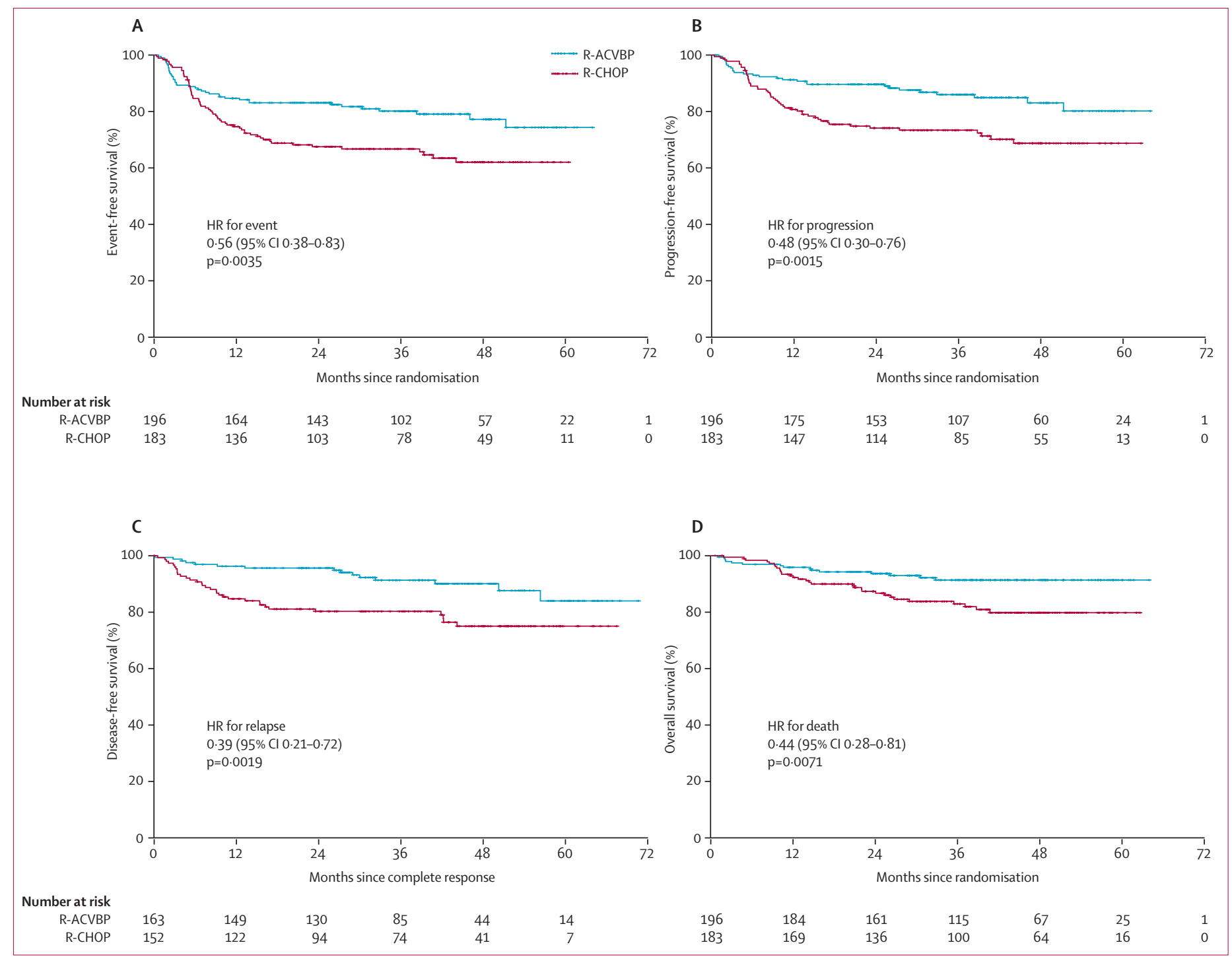

Figure 3: Kaplan-Meier estimates of outcomes by treatment group

Event-free survival for the 379 patients in the intention-to-treat population (A). Progression-free survival for the 379 patients in the intention-to-treat population (B). Disease-free survival for the 315 patients who achieved complete or unconfirmed complete response to treatment $(C)$; the number of patients in these estimates is different from the number of patients in complete or unconfirmed complete remission at the end of treatment because disease-free survival is calculated from the initial recorded response. Overall survival for the 379 patients in the intention-to-treat population (D). R-ACVBP=rituximab, doxorubicin, cyclophosphamide, vindesine, bleomycin, and prednisone. R-CHOP=rituximab, doxorubicin, cyclophosphamide, vincristine, and prednisone. $\mathrm{HR}=$ hazard ratio.

at histology review, and who received at least four cycles of the allocated treatment (165 patients in the R-ACVBP group and 156 patients in the R-CHOP group) confirmed the results of our intention-to-treat analyses: event-free survival $(\mathrm{p}=0 \cdot 0027)$, progression-free survival $(\mathrm{p}=0 \cdot 0011)$, and overall survival $(\mathrm{p}=0 \cdot 0023)$.

The presence of a bulky mass was the only factor identified as unfavourable for overall survival (HR $0 \cdot 40$, 95\% CI $0 \cdot 22-0 \cdot 72$ ) but not for progression-free survival $(0.72,0.44-1 \cdot 19)$. 3-year progression-free survival of patients with bulky disease was $77 \%$ (95\% CI 58-88) in the R-ACVBP group and 73\% (58-84) in the R-CHOP group (HR 0.69; $\mathrm{p}=0 \cdot 40$ ), and for patients without any mass larger than $10 \mathrm{~cm}$ diameter was $89 \%(83-93)$ in the R-ACVBP group and 73\% (65-85) in the R-CHOP group (HR 0.43; $\mathrm{p}=0 \cdot 0017$ ). Interactions between any baseline factor and treatment group were not significant.

The most common adverse events were haematological toxic effects in both groups (table 4). Patients receiving R-ACVBP had higher incidences of anaemia, thrombocytopenia, neutropenia, and febrile neutropenia, which were also more commonly grade 3 or 4 , than the R-CHOP group. Recorded infections during neutropenic periods were also more common in the R-ACVBP group. Grade 3 or 4 mucositis was another adverse event associated with the R-ACVBP regimen and not recorded for the R-CHOP 


\begin{tabular}{|c|c|c|}
\hline & $\begin{array}{l}\text { R-ACVBP group } \\
(n=196)\end{array}$ & $\begin{array}{l}\text { R-CHOP group } \\
(n=183)\end{array}$ \\
\hline Complete response & $114(58 \%)$ & $96(52 \%)$ \\
\hline Unconfirmed complete response & $48(24 \%)$ & $50(27 \%)$ \\
\hline Partial response & $14(7 \%)$ & $14(8 \%)$ \\
\hline Stable disease & $4(2 \%)$ & $1(1 \%)$ \\
\hline Progressive disease & $7(4 \%)$ & $17(9 \%)$ \\
\hline Death & $5(3 \%)$ & $3(2 \%)$ \\
\hline Not assessed & $4(2 \%)$ & $2(1 \%)$ \\
\hline \multicolumn{3}{|c|}{$\begin{array}{l}\text { Data are } \mathrm{n}(\%) . \mathrm{R}-\mathrm{ACVBP}=\text { rituximab, doxorubicin, cyclophosphamide, vindesine, } \\
\text { bleomycin, and prednisone. } \mathrm{R}-\mathrm{CHOP}=\text { rituximab, doxorubicin, cyclophosphamide } \\
\text { vincristine, and prednisone. Response to treatment was assessed in accordance } \\
\text { with the International Workshop } 1999 \text { criteria. }^{14}\end{array}$} \\
\hline
\end{tabular}

\begin{tabular}{|c|c|c|c|c|}
\hline & \multicolumn{2}{|l|}{ Any grade } & \multicolumn{2}{|c|}{ Grade 3 or greater } \\
\hline & R-ACVBP group & $\mathrm{R}$-CHOP group & R-ACVBP group & $\mathrm{R}-\mathrm{CHOP}$ group \\
\hline Anaemia & $194(99 \%)$ & $138(75 \%)$ & $68(35 \%)$ & $10(5 \%)$ \\
\hline Neutropenia & $189(86 \%)$ & $157(83 \%)$ & $153(78 \%)$ & $118(64 \%)$ \\
\hline Thrombocytopenia & $151(77 \%)$ & $56(31 \%)$ & $59(30 \%)$ & $6(3 \%)$ \\
\hline Febrile neutropenia & $77(39 \%)$ & $16(9 \%)$ & $75(38 \%)$ & $16(9 \%)$ \\
\hline Infection in neutropenic period & $34(17 \%)$ & $12(7 \%)$ & $26(13 \%)$ & $7(4 \%)$ \\
\hline Infection out of neutropenic period & $32(16 \%)$ & $19(10 \%)$ & $9(5 \%)$ & $5(3 \%)$ \\
\hline Mucositis & $106(54 \%)$ & $25(14 \%)$ & $35(18 \%)$ & 0 \\
\hline Nausea or vomiting & $69(35 \%)$ & $56(31 \%)$ & $6(3 \%)$ & $4(2 \%)$ \\
\hline Diarrhoea & $31(16 \%)$ & $16(9 \%)$ & $1(1 \%)$ & $1(1 \%)$ \\
\hline Cardiac-related toxic effects & $5(3 \%)$ & $1(1 \%)$ & 0 & $1(1 \%)$ \\
\hline Aminotransferase elevation & $58(30 \%)$ & $50(27 \%)$ & $4(2 \%)$ & $4(2 \%)$ \\
\hline Creatinine elevation & $11(6 \%)$ & $8(4 \%)$ & $1(1 \%)$ & 0 \\
\hline Lung-related toxic effects & $33(17 \%)$ & $15(8 \%)$ & $2(1 \%)$ & $2(1 \%)$ \\
\hline Neurological toxic effects & $50(30 \%)$ & $46(25 \%)$ & $11(6 \%)$ & $4(2 \%)$ \\
\hline Vascular toxic effects & $21(11 \%)$ & $5(3 \%)$ & $13(7 \%)$ & $2(1 \%)$ \\
\hline Rash & $51(26 \%)$ & $19(10 \%)$ & $3(2 \%)$ & 0 \\
\hline Other toxic effects & $130(66 \%)$ & $118(64 \%)$ & $25(13 \%)$ & $14(8 \%)$ \\
\hline \multicolumn{5}{|c|}{$\begin{array}{l}\text { Data are the number and proportion of patients with an event in at least one cycle. Toxic-effect grades are based on th } \\
\text { National Cancer Institute Common Terminology Criteria (version 3.0). }{ }^{15}\end{array}$} \\
\hline
\end{tabular}

group. At least one red-blood-cell transfusion was done in $99(51 \%)$ of 196 patients in the R-ACVBP group and $13(7 \%)$ of 183 in the R-CHOP group; platelet transfusions were also done in 25 (13\%) of 196 and one (1\%) of 183, respectively. Adverse events that were judged serious were more common in the R-ACVBP group (82 [42\%] of 196 patients) than in the R-CHOP group (28 [15\%] of 183). There were five deaths unrelated to lymphoma progression during the treatment period in the R-ACVBP group (three sepsis, one unknown cause, and one intrathecal vindesine injection) and three in the R-CHOP group (two sepsis and one unknown cause). At the time of our analysis, one malignancy had been recorded for the R-ACVBP group (lung cancer) and two for the R-CHOP group (biliary duct and lung cancer).

\section{Discussion}

After more than 3 years of follow-up, all our study endpoints were met, with significant improvements of event-free, progression-free, disease-free, and overall survival in patients treated with R-ACVBP.

The introduction of rituximab in association with CHOP chemotherapy has substantially improved the outcome of patients with diffuse large B-cell lymphoma. However, the results of the R-CHOP regimen, which has been widely thought the standard of care, remains suboptimum in the treatment of younger patients. In recent years, several phase 2 trials have shown promising results regarding the association of intensive chemotherapy and rituximab. ${ }^{16-19}$ Most of these regimens were proposed for patients with high-risk disease and contained an intensive chemotherapy schedule with stem-cell support. ${ }^{16-18}$ By contrast, dose-adjusted EPOCH (etoposide, prednisone, vincristine, cyclophosphamide, and doxorubicin) is an intensified regimen where pharmacodynamic dosing is on the basis of neutrophil nadir. ${ }^{19}$ A phase 2 study of dose-adjusted EPOCH plus rituximab showed promising outcomes in low and intermediate International Prognostic Index groups with progression-free survival similar to that of R-ACVBP.

ACVBP has been used in Europe to treat patients with aggressive lymphoma for the past 30 years..$^{20,21}$ The regimen consists of two different phases. The first part is an induction phase of four cycles with higher doxorubicin and cyclophosphamide doses, given at a shortened interval. During the second phase, patients receive a sequential consolidation treatment with drugs not used during induction. ACVBP improves results over CHOP in comparative trials assessing patients with localised diffuse large B-cell lymphoma ${ }^{8}$ and patients with more advanced disease. ${ }^{9}$ The association of rituximab with ACVBP in patients younger than 60 years with an ageadjusted International Prognostic Index score of 1 , as in our trial, seems to improve progression-free survival from $81 \%$ at 2 years ${ }^{22}$ to $90 \%$.

The lower rate of disease progression during the treatment phase and fewer relapses in patients who reached a complete response accounted for the prolonged progression-free survival and overall survival in our R-ACVBP group. However, we do not know which phase of the R-ACVBP regimen improves the outcome. A trial is ongoing that aims to assess the quality of remission after R-ACVBP and R-CHOP by comparing 18F-FDG PET after two and four induction cycles. ${ }^{23}$ The role of consolidation is probably important. High-dose methotrexate plus calcium folinate rescue is associated with a low risk of CNS relapse in diffuse large B-cell lymphoma. ${ }^{24}$ Ifosfamide, etoposide, and cytarabine, contained in the consolidation, are the most common drugs used in salvage regimens after relapse subsequent to anthracycline-based treatment.?

Outcome data in our patients treated with standard R-CHOP are consistent with our initial hypothesis 
(panel), ${ }^{2}$ but the 3-year event-free survival 67\% (59-73) seems to be in the lower range of the equivalent population of the MabThera International Trial: $574 \%$ (66-84) for bulk disease or 76\% (67-86) for no bulk disease. In the MabThera International Trial, various chemotherapy regimens plus rituximab did not produce major differences in outcome. However, $40 \%$ of the patients treated with the rituximab combination received radiotherapy. Whether radiotherapy could affect the outcome in this population treated with R-CHOP is being studied at present by the Deutsche Studiengruppe fur Hochmaligne Non-Hodgkin-Lymphome (registered with ClinicalTrials.gov, number NCT00278408).

The profile of toxic effects for the R-ACVBP regimen is well characterised. The induction phase is associated with noticeable haematological toxic effects, which is why the regimen is only used in patients younger than 60 years. ${ }^{9}$ Most patients experience substantial neutropenia between day 9 and day 13 of each cycle, with a substantial proportion (39\%) having a febrile episode despite the use of growth factor. However, our trial and previous ones involving the ACVBP regimen ${ }^{25}$ have shown that lifethreatening complications related to treatment are uncommon in a population of patients younger than 60 years with good performance status. The care of many patients included in our trial has been managed in the oncology departments of community hospitals of the 73 participating centres from the GELA network. Although ACVBP is an intensified regimen, the total dose of doxorubicin and cyclophosphamide given is less than the dose received during eight cycles of CHOP. This dose might account for the somewhat low incidence of secondary malignancies and cardiac-related toxic effects recorded in a large cohort of 2837 ACVBP-treated patients that were followed up over a median of 74 months. ${ }^{26} \mathrm{We}$ plan to do long-term follow-up of our patient cohort.

In the context of chemotherapy, interactions between the microenvironment and cancer cells, especially lymphoma cells, are complex. ${ }^{27}$ There is some evidence that antibody-dependent cellular cytotoxicity mediated by activation of natural-killer cells contributes to the clinical response to rituximab. ${ }^{28}$ Investigators have postulated that intensive chemotherapy could compromise the efficacy of rituximab by impairing immune cellular mechanisms. ${ }^{5}$ However, the results of our study do not favour such a hypothesis.

Modifications of immunochemotherapy regimens are still worthwhile and are being assessed at present. In our selected population, we did not identify any factor to suggest that a specific patient category would benefit from treatment over the other. The favourable results obtained with R-ACVBP front-line regimen should be further assessed in view of the biological heterogeneity of diffuse large B-cell lymphoma, ${ }^{29}$ to assess whether some patients with specific subtypes are more to likely to benefit from this intensive therapy, as it has been suggested for doseadjusted EPOCH plus rituximab, ${ }^{19}$ whereas R-CHOP
Panel: Research in context

\section{Systematic review}

We searched Medline from January, 2002, to May, 2011, for full papers reporting randomised clinical trials with the terms "rituximab" and "diffuse large B-cell lymphoma". We identified four randomised clinical trials comparing an association of chemotherapy and rituximab versus chemotherapy alone in patients given first-line treatment for diffuse large B-cell lymphoma. ${ }^{2,4-6}$ Only one of these trials has been done in patients younger than 60 years. ${ }^{5}$ We did not identify any clinical trial that compared two chemotherapy regimens combined with rituximab.

\section{Interpretation}

These studies have established the addition of the anti-CD20 monoclonal antibody rituximab with a chemotherapy regimen of cyclophosphamide, doxorubicin, vincristine, and prednisone (CHOP) as a new standard of care. However, $\mathrm{CHOP}$ plus rituximab is suboptimum for a substantial number of patients who could relapse and are not salvaged by subsequent therapies. Our results show that rituximab combined to the intensified multidrug regimen of doxorubicin, cyclophosphamide, vindesine, bleomycin, and prednisone (ACVBP), as first-line treatment, improves the survival of young patients with low-intermediate-risk diffuse large B-cell lymphoma. Haematological and mucosal toxic effects induced by ACVBP plus rituximab are raised but manageable in a multicentre setting.

might be sufficient for others. The possibility of decreasing the intensity of immunochemotherapy could also be explored in selected patients who show a rapid response to treatment, assessed with interim PET. Pending these results and the advent of new targeted therapies in frontline treatment, intensified immunochemotherapy with R-ACVBP represents an alternative to R-CHOP to improve survival in patients younger than 60 years with diffuse large B-cell lymphoma of low-intermediate risk.

\section{Contributors}

CR, BCoi, CH, TJM, AB, J-PJ, GS, and HT designed the study. CR, BC $\mathrm{CH}, \mathrm{CFer}, \mathrm{OC}, \mathrm{CT}, \mathrm{AB}, \mathrm{GL}, \mathrm{FM}, \mathrm{HG}, \mathrm{FJ}, \mathrm{SB}, \mathrm{CFru}$, BCor, JG, CB, MJ and HT collected data. TJM and DC were responsible for the histological review. CR, BCoi, CH, J-PJ, GS, and $\mathrm{HT}$ analysed and interpreted the data and wrote the report. All authors had full access to the final version of the report and agreed to the submission.

\section{Trial investigators}

Belgium A Van Hoof (Academisch Ziekenhuis Sint Jan AV, Brugge); K Van Eygen (Academisch Ziekenhuis Groeninge, Coutrai); S Lamperz (Centre Hospitalier Bois de l'Abbaye, Seraing); M André (Centre Hospitalier Notre Dame et Reine Fabiola, Charleroi); C Bonnet, G Fillet (Centre Hospitalier Universitaire, Liège); D Boulet (Clinique Saint Joseph, Mons); M Maerevoet (Clinique Saint Pierre, Ottignies); E Van Den Neste (Cliniques Universitaires Saint Luc, Louvain); A Bosly (Cliniques Universitaires de Mont Godinne, Mont Godinne); H Demuynck (Heilig Hart Ziekenhuis, Leuven); N Straetmans (Hôpital Jolimont, Haine Saint Paul); V Mathieux (Hôpital Sainte Elisabeth, Namur); P Mineur (Hôpital Saint Joseph, Gilly); P Pierre (Hôpital Saint Joseph, Arlon); D Bron (Institut Jules Bordet, Brussels); A Kentos (Université Libre de Bruxelles, Hôpital Erasme, Brussels); P Zachee (ZNA Stuivenberg, Antwerpen). France C Fruchart (Centre François Baclesse, Caen); G Marit (Centre François Magendie, Bordeaux); F Jardin, H Tilly (Centre Henri Becquerel, Rouen); B Corront, C Martin (Centre Hospitalier, Annecy); G Lepeu (Centre Hospitalier, Avignon); H Orfeuvre (Centre Hospitalier, Bourg en Bresse); B Salles (Centre Hospitalier, Châlon sur Saone); M Blanc (Centre Hospitalier, Chambéry); L Al Jassem (Centre Hospitalier, Chartres); M Wetterwald (Centre Hospitalier, Dunkerque); C Kulekci (Centre Hospitalier Marc Jacquet, Melun); X Vallantin (Centre Hospitalier Maréchal Joffre, Perpignan); 
C Soussain (Centre Hospitalier, Meaux); J C Eisenmann (Centre Hospitalier, Mulhouse); F Morvan (Centre Hospitalier René Dubos, Pontoise); M Azagury (Centre Hospitalier, Saint Germain en Laye); A Devidas (Centre Hospitalier Sud Francilien, Corbeil); F Morschhauser (Centre Hospitalier Universitaire, Lille); S Bologna, P Feugier (Centre Hospitalier Universitaire, Nancy); M Macro (Centre Hospitalier Universitaire, Caen); O Casanovas (Centre Hospitalier Universitaire, Dijon); D Bordessoule (Centre Hospitalier Universitaire, Limoges); J M Karsenti, N Mounier (Centre Hospitalier Universitaire, Nice); E Jourdan (Centre Hospitalier Universitaire, Nîmes); R Herbrecht (Centre Hospitalier Universitaire, Strasbourg); G Laurent, C Récher (Centre Hospitalier Universitaire, Toulouse); B Coiffier, G Salles (Centre Hospitalier Universitaire Lyon-Sud, Pierre-Bénite); A Delmer (Centre Hospitalier Universitaire, Reims); B Anglaret (Centre Hospitalier, Valence); H Ghesquiéres, C Sebban (Centre Léon Bérard, Lyon); M Janvier (Centre René Huguenin, Saint Cloud); M Fabbro (Centre Val d'Aurelle, Montpellier); M Flesch (Fondation Drevon, Dijon); S Castaigne (Hôpital André Mignot, Versailles); P Fenaux (Hôpital Avicenne, Bobigny); C Besson (Hôpital Bicêtre, Le Kremlin-Bicêtre); L Mosser (Hôpital Bourran, Rodez); B Christian (Hôpital Bon Secours, Metz); J Gabarre (Hôpital de la Pitié Salpétriere, Paris); F Maréchal (Hôpital des Chanaux, Macon); T De Revel (Hôpital d'Instruction des Armées Percy, Clamart); K Belhadj, C Haioun (Hôpital Henri Mondor, Créteil); Z Marjanovic (Hôpital Hôtel Dieu, Paris); O Fain (Hôpital Jean Verdier, Bondy); R Delarue (Hôpital Necker, Paris); G Sebahoun (Hôpital Nord, Marseille); B Audhuy (Hôpital Pasteur, Hôpitaux Civils, Colmar); M Aoudjhane (Hôpital Saint Antoine, Paris); C Gisselbrecht, C Thièblemont (Hôpital Saint-Louis, Paris); C Rose (Hôpital Saint Vincent de Paul, Lille); E Fleck (Hôpital Saint-Louis, La Rochelle); D Decaudin (Institut Curie, Paris); P Colin (Institut de Cancérologie Courlancy, Reims); C Fermé, V Ribrag (Institut Gustave Roussy); O Fitoussi (Polyclinique Bordeaux Nord Aquitaine, Bordeaux). Switzerland N Ketterer (Centre Hospitalier Vaudois, Lausanne).

\section{Conflicts of interest}

BCoi, CH, OC, AB, GL, FM, GS, and HT have served as consultants, on advisory boards, or both for Roche, Genentech, or both. CR, GS, and HT have served as consultants, on advisory boards, or both for Celgene. CR is an advisory board member of Genzyme and received grant support from Celgene. BCoi received grant support from Amgen and payment for lectures from Amgen, Celgene, Roche, and GlaxoSmithKline. $\mathrm{CH}$ is an advisory board member of Pfizer and Janssen-Cilag, received grant support from Amgen, Celgene, and Roche, and received payment for lectures from Roche. OC received grants support from Amgen, Chugai, and Roche. AB received grant support from Roche and received payment for lectures from Roche. FM received payment for lectures from Bayer, Novartis, and Roche. GS received payment for lectures from Celgene, Mundi Pharma, and Roche, and received travel support from Pfizer. HT is an advisory board member of Seattle Genetics, received grant support from Amgen and Celgene, received payment for lectures from Amgen, Celgene, Janssen-Cilag, and Pfizer, and travel support from Celgene. TJM, CFer, CT, HG, FJ, SB, CFru, BCor, JG, CB, MJ, DC, and J-PJ declare that they have no conflicts of interest.

\section{Acknowledgments}

We thank the pathologists participating in the histological review: J Brière, D Canioni, C Copie-Bergman, J Diebold, J F Emile, B Fabiani, P Gaulard, T J Molina, and T Petrella. We thank the staff of the GELARC for the management of this work.

\section{References}

1 Morton LM, Wang SS, Devesa SS, Hartge P, Weisenburger DD, Linet MS. Lymphoma incidence patterns by WHO subtype in the United States, 1992-2001. Blood 2006; 107: 265-76.

2 Coiffier B, Lepage E, Briere J, et al. CHOP chemotherapy plus rituximab compared with CHOP alone in elderly patients with diffuse large-B-cell lymphoma. N Engl J Med 2002; 346: 235-42.

3 Coiffier B, Thieblemont C, Van Den Neste E, et al. Long-term outcome of patients in the LNH-98.5 trial, the firs randomized study comparing rituximab-CHOP to standard CHOP chemotherapy in DLBCL patients: a study by the Groupe d'Etudes des Lymphomes de l'Adulte. Blood 2010; 116: 2040-45.
4 Habermann TM, Weller EA, Morrison VA, et al. Rituximab-CHOP versus CHOP alone or with maintenance rituximab in older patients with diffuse large B-cell lymphoma. J Clin Oncol 2006; 24: 3121-27.

5 Pfreundschuh M, Trumper L, Osterborg A, et al. CHOP-like chemotherapy plus rituximab versus CHOP-like chemotherapy alone in young patients with good-prognosis diffuse large-B-cell lymphoma: a randomised controlled trial by the MabThera International Trial (MInT) Group. Lancet Oncol 2006; 7: 379-91.

6 Pfreundschuh M, Schubert J, Ziepert M, et al. Six versus eight cycles of bi-weekly CHOP-14 with or without rituximab in elderly patients with aggressive CD20+ B-cell lymphomas: a randomised controlled trial (RICOVER-60). Lancet Oncol 2008; 9: 105-16.

7 Gisselbrecht C, Glass B, Mounier N, et al. Salvage regimens with autologous transplantation for relapsed large B-cell lymphoma in the rituximab era. J Clin Oncol 2010; 28: 4184-90.

8 Reyes F, Lepage E, Ganem G, et al. ACVBP versus CHOP plus radiotherapy for localized aggressive lymphoma. N Engl J Med 2005 352: 1197-205.

9 Tilly H, Lepage E, Coiffier B, et al. Intensive conventional chemotherapy (ACVBP regimen) compared with standard CHOP for poor-prognosis aggressive non-Hodgkin lymphoma. Blood 2003; 102: 4284-89.

10 The International Non-Hodgkin's Lymphoma Prognostic Factors Project. A predictive model for aggressive non-Hodgkin's lymphoma. N Engl J Med 1993; 329: 987-94.

11 Pfreundschuh M, Trumper L, Kloess M, et al. Two-weekly or 3-weekly CHOP chemotherapy with or without etoposide for the treatment of young patients with good-prognosis (normal LDH) aggressive lymphomas: results of the NHL-B1 trial of the DSHNHL. Blood 2004; 104: 626-33.

12 Swerdlow SH, Campo E, Harris NL, et al. World Health Organization classification of tumours of haematopoietic and lymphoid tissues. Lyon: IARC Press, 2008.

13 Oken MM, Creech RH, Tormey DC, et al. Toxicity and response criteria of the Eastern Cooperative Oncology Group. Am J Clin Oncol 1982; 5: 649-55.

14 Cheson BD, Horning SJ, Coiffier B, et al. Report of an international workshop to standardize response criteria for non-Hodgkin's lymphomas. NCI Sponsored International Working Group. J Clin Oncol 1999; 17: 1244-50.

15 National Cancer Institute. Common terminology criteria for adverse events v3.0. http://ctep.cancer.gov/protocolDevelopment/ electronic_applications/docs/ctcaev3.pdf (accessed Oct 11, 2011).

16 Tarella C, Zanni M, Di Nicola M, et al. Prolonged survival in poor-risk diffuse large B-cell lymphoma following front-line treatment with rituximab-supplemented, early-intensified chemotherapy with multiple autologous hematopoietic stem cell support: a multicenter study by GITIL (Gruppo Italiano Terapie Innovative nei Linfomi). Leukemia 2007; 21: 1802-11.

17 Vitolo U, Chiappella A, Angelucci E, et al. Dose-dense and high-dose chemotherapy plus rituximab with autologous stem cell transplantation for primary treatment of diffuse large B-cell lymphoma with a poor prognosis: a phase II multicenter study. Haematologica 2009; 94: 1250-58.

18 Glass B, Ziepert M, Reiser M, et al. High-dose therapy followed by autologous stem-cell transplantation with and without rituximab for primary treatment of high-risk diffuse large B-cell lymphoma. Ann Oncol 2010; 21: 2255-61.

19 Wilson WH, Dunleavy K, Pittaluga S, et al. Phase II study of dose-adjusted EPOCH and rituximab in untreated diffuse large B-cell lymphoma with analysis of germinal center and post-germinal center biomarkers. J Clin Oncol 2008; 26: 2717-24.

20 Coiffier B, Bryon PA, Berger F, et al. Intensive and sequential combination chemotherapy for aggressive malignant lymphomas (protocol LNH-80). J Clin Oncol 1986; 4: 147-53.

21 Coiffier B, Gisselbrecht C, Herbrecht R, Tilly H, Bosly A, Brousse N. LNH-84 regimen: a multicenter study of intensive chemotherapy in 737 patients with aggressive malignant lymphoma. J Clin Oncol 1989; 7: 1018-26.

22 Morel P, Gaulard P, Gisselbrecht C, et al. Autologous stem-cell transplantation as consolidation therapy for diffuse large B-cell lymphoma patients with overexpression of bcl-2 protein. Results of the Groupe d'Etude des Lymphomes de l'Adulte (GELA) trial LNH98-B2. Ann Oncol 2008; 19: 560-65. 
23 Casasnovas RO, Meignan M, Berriolo-Riedinger A, et al. SUVmax reduction improves early prognosis value of interim positron emission tomography scans in diffuse large B-cell lymphoma. Blood 2011; 118: 37-43.

24 Abramson JS, Hellmann M, Barnes JA, et al. Intravenous methotrexate as central nervous system (CNS) prophylaxis is associated with a low risk of CNS recurrence in high-risk patients with diffuse large B-cell lymphoma. Cancer 2010; 116: 4283-90.

25 Dumontet C, Mounier N, Munck JN, et al. Factors predictive of early death in patients receiving high-dose CHOP (ACVB regimen) for aggressive non-Hodgkin's lymphoma: a GELA study. Br J Haematol 2002; 118: 210-17.
26 André M, Mounier N, Leleu X, et al. Second cancers and late toxicities after treatment of aggressive non-Hodgkin lymphoma with the ACVBP regimen: a GELA cohort study on 2837 patients. Blood 2004; 103: 1222-28.

27 Gilbert LA, Hemann MT. DNA damage-mediated induction of a chemoresistant niche. Cell 2010; 143: 355-66.

28 Weiner GJ. Rituximab: mechanism of action. Semin Hematol 2010; 47: 115-23.

29 Lenz G, Staudt LM. Aggressive lymphomas. $N$ Engl J Med 2010; 362: 1417-29. 